\title{
Diagnostic utility of digital cholangioscopy for dislodged bile duct tumor thrombus of hepatocellular carcinoma
}

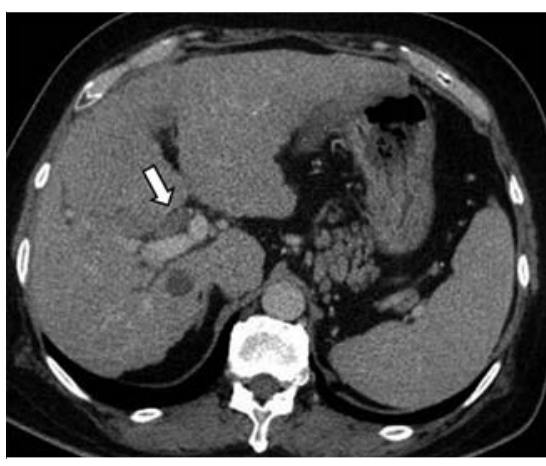

- Fig. 1 Computed tomography revealed an intraductal tumor lesion (arrow) from the right hepatic duct to the common hepatic duct, with intrahepatic bile duct dilatation. Liver cirrhosis and liver cyst were also detected, with absence of hepatic tumor.

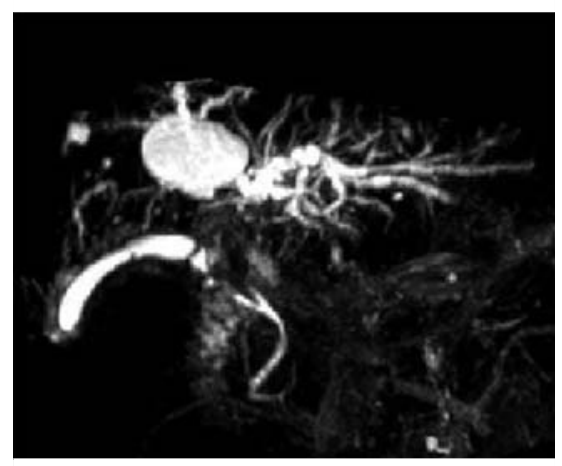

- Fig. 2 Magnetic resonance cholangiopancreatography revealed hilar bile duct stricture with intrahepatic bile duct dilatation.

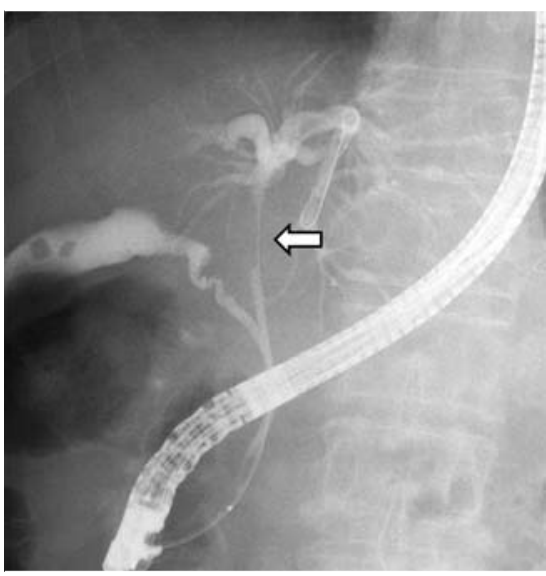

- Fig. 3 Cholangiography revealed hilar bile duct stricture (arrow). The right hepatic duct was not detected because of severe stricture, and the stricture had reached the medial branch bifurcation in the left hepatic duct.

In the common hepatic duct stricture, a mold-like tissue fragment was seen within the bile duct lumen with regular bile duct wall ( $>$ Video 1 ); removal was attempted by using basket and balloon
Bile duct tumor thrombus (BDTT) of hepatocellular carcinoma (HCC) is rare, and making a differential diagnosis from bile duct cancer is often challenging [1]. We present a rare case of HCC with BDTT mimicking hilar bile duct cancer, which could be diagnosed by a new digital cholangioscopy system (SpyGlass DS; Boston Scientific Corp., Marlborough, Massachusetts, USA) [2].

A man in his 60s was admitted because of jaundice. His medical history was unremarkable. Liver enzyme and bilirubin levels were elevated. Computed tomography revealed an intraductal tumor lesion from the right hepatic duct to the common hepatic duct, with intrahepatic bile duct dilatation ( $\triangleright$ Fig. 1). Liver cirrhosis and liver cyst were also detected, without hepatic tumor. Magnetic resonance cholangiopancreatography revealed hilar bile duct stricture ( $\triangleright$ Fig. 2 ). Endoscopic retrograde cholangiography revealed that the stricture had reached the medial branch bifurcation in the left hepatic duct, and the right hepatic duct could not be detected because of severe stricture ( $\triangleright$ Fig. 3). Hilar bile duct cancer was initially considered based on these findings. However, intraductal ultrasonography revealed that the bile duct wall was regular; thus, cholangioscopy was performed. 


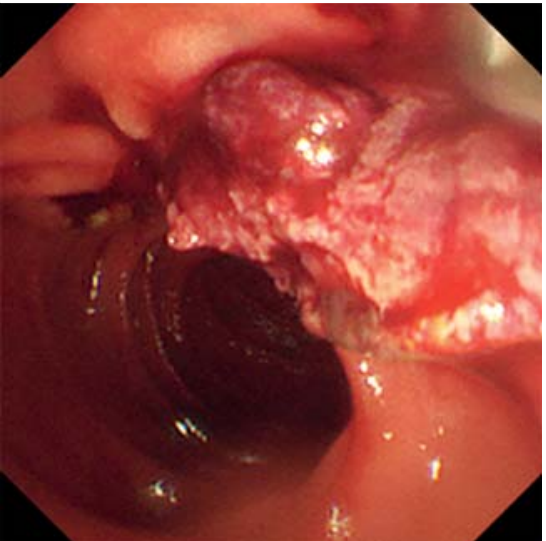

- Fig.4 Endoscopic view showing tissue fragment removed from the bile duct.

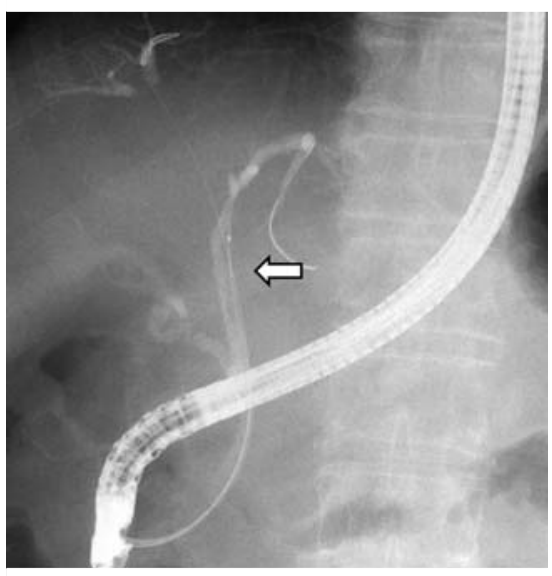

Competing interests

None

The Authors

Tadahisa Inoue, Tomohiko Ohashi, Yukiomi Nakade, Yuji Kobayashi, Norimitsu Ishii, Kiyoaki Ito, Masashi Yoneda Department of Gastroenterology, Aichi Medical University School of Medicine, Nagakute, Japan
Corresponding author

\section{Tadahisa Inoue, MD}

Department of Gastroenterology, Aichi Medical University School of Medicine,

1-1 Yazakokarimata, Nagakute,

Aichi 480-1195, Japan

Fax: +81-561-633208

tinoue-tag@umin.ac.jp

\section{References}

[1] Wu Z, Guo K, Sun H et al. Caution for diagnosis and surgical treatment of recurrent cholangitis: lessons from 5 cases of bile duct tumor thrombus without a detectable intrahepatic tumor. Medicine 2014; 93: e80

[2] Navaneethan U, Hasan MK, Kommaraju K et al. Digital, single-operator cholangiopancreatoscopy in the diagnosis and management of pancreatobiliary disorders: a multicenter clinical experience (with video). Gastrointest Endosc 2016; 84: 649-655

\section{Bibliography}

DOI http://dx.doi.org/10.1055/s-0042-121007

Endoscopy 2017; 49: E33-E34

(C) Georg Thieme Verlag KG

Stuttgart · New York

ISSN 0013-726X
Fig. 5 After endoscopic biliary extraction and drainage, no stricture was detected from the left hepatic duct to the common hepatic duct (arrow), but the right hepatic duct stricture persisted. 